\title{
Les Causes de Délinquence et La Responsabilité Pénale Du Fait Des Activités Sportives ${ }^{\bullet}$
}

\author{
Assoc. Prof.Dr.iur. Pınar Memiş Kartal ${ }^{\bullet \bullet}$
}

\begin{abstract}
Violence and penal liability arising from sport activities are some of the most controversial subjects in criminal law. The importance of sport is primarily in its economic power. This article briefly covers the main sub-titles of reasons giving rise to violence in sporting activities and penal liability arising from violent actes resulting from such reasons related to sports as a multidisciplinary field.
\end{abstract}

Key words: Sports, violence in sports, match fixing, doping, corruption

\begin{abstract}
Le sport est une activité le plus ancien et aussi le plus populaire du monde. De nos jours, les causes de délinquance dans le sport et la responsabilité pénale du fait des activités sportives est l'un des sujets le plus populaire. C'est parce que le sport est une activité ludique, un pouvoir économique. En tant qu'une activité multi-disciplinaire, notre article sera sur cet évenement populaire qui cause aussi la violence et la responsabilité pénale.
\end{abstract}

Les mots clés: Sport, La violence dans le sport, le match fixé, le doping, la corruption

Le sport est une activité de participation, d'appartenance, de revendication et d'intégration à la société ${ }^{1}$. Aujourd'hui la place et l'importance du sport ne sont plus contestées. Le sportif en adhèrant à cette activité gagne une possibilité de se prononcer à sa façon, une identité, une culture et aussi parfois un salaire. Il n'ya pas seulement le sportif dans cette activité, il ya des hommes de sport comme l'antreneur, le directeur technique, l'organisateur, l'arbitre, le médecin, les organisations comme les clubs, les fédérations, et sans oublier les spectateurs qui sont un des points le plus important dans l'activité sportive. La place du sport est aussi importante en droit turc. La constitution Turque 1982 prévoit dans son article 59 le sport par son titre "promotion du sport" en disant "L'Etat prend les mesures propres à améliorer la santé physique et mentale des citoyens turcs de tout âge et encourage l'extension de la pratique du sport par les masses.L'Etat protège les sportifs méritants En ce qui concerne les décisions des fédérations sportives relatives à la gestion et la discipline des activités sportives, on peut recourir à l'arbitrage obligatoire. Les décisions prises par le tribunal arbitral sont définitives at aucune autorité judiciaire ne peut être saisie. (intitulé modifié at alinéa 3 ajouté par la loi no: 6214 du 17 mars 2011)».Avec tous ces élements le sport est une activité ludique, intellectuelle et compétitive et aussi un spectacle qui tient une place importante dans l'espace économique de chaque pays. Cette place importante du sport entraine aussi des inconvenients, des enjeux et des risques qui peuvent conduire à la tragédie. Il faut donc constater ici, l'antagonisme du fait de l'évenement sportif qui fait cohabiter pureté et impureté, norme et déviance $^{2}$. Parlant de l'antagonisme, la partie tragique du sport nous emmene aux crimes ou plutôt des délits. Nous pouvons constater les crimes ${ }^{3}$ prévues d'une part, dans le Code Pénal comme l'homicide par négligence (Code pénal Turc art.85), les lésions corporelles ou les voies de fait intentionnels ou par négligence (on utilise le

\footnotetext{
- Cette étude est effectuée avec le soutien de la Commission des recherches scientifiques de l'Université Galatasaray.

'Professeur associé au département de droit pénal et de procédure pénale de la Faculté de Droit à l'Université de Galatasaray. Notre objectif est de présenter une partie de résultat d'une recherce scientifique et de remercier pour le financement à la Comission De Projet de Recherche Scientifique De l'Université de Galatasaray.
}

${ }^{1}$ MOREILLON Laurent, Introduction-Vers un nouveau droit pénal du sport, in Aspects pénaux du droit du sport, Collection CIES, Staempfli Editions SA Berne.2002, p.7 (p.7-14).

${ }^{2}$ BOUCHET Patrick/CASTEL Philippe/LACASSAGNE Marie-Françoise, "Comment analyser les relations déviantes potentiellement violentes ou discriminatoires dans le spectacle sportif au stade?”, in Sport Science Review, vol. XX, No. 1-2, April 2011, pp. 139 (pp. 137-165). DOI: 10.2478/v10237-011-0051-6.

${ }^{3}$ ÖZBEK Veli Özer/KANBUR Nihat/DOĞAN Koray/ BACAKSIZ Pınar/TEPE İlker; Türk Ceza Hukuku Özel Hükümler, Seçkin, Eylül 2014.” 
même terme en droit pénal turc, (CPT.art.86-89), l'injure (CPT. art. 125), la discrimination (CPT. art. 122) et d'autre part les faits déviants de nature purement sportive comme doping ${ }^{4}$ qui n'est pas connu comme un délit dans le code mais un fait illégal contre fair play connu par les réglements sportifs des Fédérations; les faits de violence connus par la Loi 6222 de "Lutte contre la violence et le désordre dans le sport"; la corruption qui est la tricherie en vers le skor du match ou de compétion sportive. La violence dans le sport comprend bien sur l'homicide, les voies de fait mais comme dans les réglementations du Conseil de l'Europe, on parle ici d'une violence dite sportive comme hooliganisme, porter atteinte a l'intégrité corporelle des hommes de sport et aussi les actes contre les biens de l'environnement du sport comme le fait jeter les pierres ou les bouteilles au stade etc.. Dans les faits cités il y'en a d'entre eux qui sont à la fois prévus par la Loi 6222 et les réglements des Fédérations sportives et il y'en a qui sont prévus simplement par les réglements qui sont considérés moins importants et moins dangeureux. Au-delà de l'affirmation des faits, il nous faut examiner les types de délinquance causés par les hommes du sport comme le sportif, le spectateur, l'organisateur etc. Chacun a le droit de pratiquer de l'activité sportive qui lui convient mais bien sur avec le respect aux régles des principes. Par cette perspective, pour pouvoir comprendre les raisons de la tragédie dans le sport, il faut montrer les facteurs de la délinquance dans le sport. Par la compréhension des facteurs on peut échapper des délits. Mais une fois qu'un acte dit délit ou crime soit commis, il faut préciser la responsabilité pénale du fait des activités sportives en droit ${ }^{5}$, surtout en droit pénal Turc.

\section{LES CAUSES DE DÉL $\square$ NQUANCES DANS LE SPORT}

Les causes de délinquance dans les sports sont très variées et parfois change d'après la nature du sport. La variance dépend principalement de la société, de son histoire et c'est la raison pour la quelle la détermination des causes de délinquance dans le sport est un des problématiques des sociologues et des criminologues. Comme disait NUYTENS en décrivant la violence sportive, "la violence se traduit de manière différentes en fonction de la dynamique des groupes, de leur histoire rythmée par des contentieux, du rôle et de la position occupée par le ou les supporters violents" ${ }^{\text {. }}$ Les causes de délinquances non seulement la violence mais pour les autres faits illégals aussi, il faut au moins démontrer les points de repères. Notre objectif pour ce travail, est de montrer les causes reconnues et générales pour toute sorte des sports. En effet, il semble qu'il ya un multiple des comportements déviants, violents ou discriminatoires dans le milieu sportifs ${ }^{7}$.Comme dans toutes sortes de criminalité, en criminalité sportive on constate que la premiere raison de délinquance est la société. La nature, la culture et la perception sociale est l'une des facteurs de la criminalité sportive. Par exemple la plupart des cas de violence fait provoquer par les goupes de meme classe sociale. Donc la société est le point de départ des causes de délinquance dans le sport.L'aspect économique du phénomène est la deuxième raison de la criminalité dans le sport. Pour tous les cas de délinquaces dites sportive comme la violence, le doping, la tricherie, la corruption, on constate que le facteur économique est le plus important. Du sportif au spectateur, les participants à l'activité sportive, les parieurs, le peuple du monde sportif veut profiter de cet acte qui est à la fois spectacle, à la fois business.La victoire est l'une des facteurs de délinquance en sport. La réussite dans le milieu sportif entraine un pouvoir et un nouveau mode de vie et aussi la chance du choix entre les meilleurs clubs. C'est la raison pour la quelle un sportif n'hésite pas d'utiliser la substance ou les méthodes en question dite doping ${ }^{8}$.Nous pouvons multiplier les facteurs ou les raisons de délinquance dans le milieu sportif mais tous vont être les dérivées de ce qu'on a cité. L'homme dépendant de sa classe sociale, veut gagner quelque soit l'argent, la victoire, en faite un statut économique élevé. Ce la s'explique par le coté ludique du sport ${ }^{9}$. Tout cela est lié bien à la psychologie de la personne. Les parieurs veulent gagner un peu plus, l'organisateur veut profiter de son organisation, le sportif veut la réussite et tout cela entraine d'abord la faute au fair play puis les crimes.Dans ce cas là, il faut se demander comment lutter avec ce phénomène? Chaque pays qui participe aux organisations sportives nationales ou internationales a ses propres codifications comme la Turquie. C'est la raison pour la quelle dans la deuxième partie de cette présente contribution, nous voulons aborder le problème de la responsabilité pénale du fait des activités sportives en cas de commission des crimes par les agents sportifs d'après le droit pénal turc.

\footnotetext{
${ }^{4}$ ERKİNER Kısmet, Hukuk Boyutunda Doping, Nobel Yayın Dağıtım, Ankara Şubat 2006, p. 231.

${ }^{5}$ SCHILD Wolgang, Antrenörün, Hakemin ve Seyircinin Ceza Sorumluluğu, çeviren: HAKERİ Hakan, in: "Spor ve Ceza Hukuku"-Karşılaştırmalı Güncel Ceza Hukuku Serisi 1, Seçkin, Ankara 2004, pp. 41-59.

${ }^{6}$ NUYTENS Williams, "La violence dans les stades de football. Eléments d'étiologie à partir du cas des autonomes du Racing Club de Lens", in: Revue internationale de Criminologie et de Police technique et scientifique, Volume LV, No:3, 2002, Juillet-Septembre, p. 295 (pp.277-300).

${ }^{7}$ BOUCHET/CASTEL/LACASSAGNE,Comment analyser les relations déviantes potentiellement violentes ou discriminatoires dans le spectacle sportif au stade, p. 144. ;

${ }^{8}$ MIMIAGUE Marie-Jose, "Le Problème du Doping en Droit Pénal Comparé", Thèse pour le doctorat en Droit présentée et soutenue le 16 Juin 1973, Université de Bordeaux I, sh. 8-9.

${ }_{9}^{9}$ DEFRANCE Jacques, Sociologie du sport, La DECOUVERTE, Paris 1995, p. 11 ets.. 


\section{LA RESPONSAB $\square$ L $\square$ TÉ PÉNALE DU FA $\square$ T DES ACT $\square$ V $\square$ TÉS SPORT $\square$ VES}

La responsabilité pénale du fait des activités sportives est un sujet nouveau dans le droit pénal turc. La nouveauté n'est pas de connaitre les actes sportif comme les faits justificatifs, ce qui est nouveau, c'est de définir les crimes dites sportives comme la corruption, les matchs fixés, les paris sportifs truqués, les actes de violence contre les biens sportifs etc.. Le doping aussi est une sorte de corruption ${ }^{10}$ mais il n'est pas considéré comme un acte criminel mais un acte illégal prévu par les Réglements disciplinaires des Fédérations. Pourtant l'usage des substances ou des méthodes considéré à la fois drogue, à la fois doping, dans ce cas là le fait est considéré un acte criminel prévu dans le code pénale. Ces actes de nature sportive, c'est à dire les actes qui peuvent se faire seulement à l'occasion d'une compétition sportive ont été abordé et déterminé par une loi en 2011 par la loi 6222. Jusqu' à cette loi édite, il n'existait pas de règles pénales spécifiques en Turquie qui concernent les délits commis par les hommes sportifs, les spectateurs. Dans ce travail, on ne va pas aborder en détail le problème du fait justificatif mais on veut essayer de montrer les actes sportifs déterminés comme crime; la raison de les déterminer comme crime et la responsabilité pénale du fait de ces activités dites sportives ${ }^{11}$ mais en réalité non-sportives, contraire au fair play. Comme LAPOUBLE cite dans son livre "“les valeurs sportives"marquent le pas face aux valeurs fondatrices des régimes démocratiques"12.

\section{Jusitification du régime de résponsabilité pénale}

Comme nous l'avions remarqué qu'il n'existait pas de règles pénales spécifiques en droit turc qui concernent les délits commis par des sportifs, ce qui signifie que les sportifs doivent être soumis aux dispositions pénales ordinaires. Cela cause aussi l'intervention minimale du juge pénale aux affaires sportifs ${ }^{13}$. Il faut donc souligner que seules les infractions commises par négligence ou celles de peu de gravité pourront tomber sous le coup de l'immunité. Il faudra alors se demander les risques et les dangers inhérents à un sport déterminé sont tels qu'ils dépassent les bienfaits qu'on peut raisonnablement en attendre. Pour resoudre ce probléme, il faut examiner les cas par le principe du consentement de la victime, le principe de l'activité autorisée, l'acceptation des risques et la permission du droit.Le fameux principe du consentement de la victime (volenti non fit injuria) dépend du consentement du sportif qui participera à la compétition mais il faut bien dire qu'aujourd'hui, cette thèorie est rejeté par la plupart de la doctrine ${ }^{14}$. Quant au principe de l'activité autorisée, ceci est plus délicat par rapport au consentement dela victime puis que l'activité est autorisée par des législations. La base de références est juridique. Comme nous l'avions précité d'abord la Constitution, les lois des organisations sportives et ses textes, ses réglements etc. En effet, le risque accepté non plus n'exclut pas la possibilité de sanctionner la maladresse ou l'imprévoyance et, à fortiori, la volonté de nuire, c'est pourquoi a été condamné un joueur de football coupable de brutalités prohibées par les règles du jeu. En revanche, le fait pour un footballeur de fracturer, au cours d'une partie, la jambe d'un adversaire ne constitue pas le délit de blessures involontaires dès lors que, dans la phase de jeu au cours de laquelle s'est produit l'accident, il a, sans qu'il puisse lui être reproché une imprudence ou une négligence, pratiqué un acte autorisé par les règles du sport.Dans les sports qui ne comportent pas précisément de violence mais qui présentent un certain nombre de dangers, comme les sports de montagne, l'imprudence ou l'imprévoyance peuvent également être retenues par les tribunaux. Le ski de fond ou l'escalade comportent, des risques objectifs (tempêtes, chute de pierres) ou subjectifs (chutes). Mais si le skieur ou l'alpiniste, se fiant aux conseils d'un guide, subit un dommage par la faute, l'imprudence ou l'imprévoyance de celui-ci, les tribunaux en se livrant à une appréciation in concreto de la situation peuvent retenir la responsabilité pénale.En revanche, lorsque le sport n'est ni violent ni dangereux par nature, les décisions de justice peuvent être sévères. La seule réalisation d'un dommage suffit alors à caractériser l'imprévoyance, cause du préjudice. La finalité de la jurisprudence est, ici, d'assurer au mieux la réparation du préjudice de la victime. En effet, il n'est pas exagéré d'affirmer qu'il n'y a pas à proprement parler de droit de la responsabilité sportive. C'est bien au droit commun de la responsabilité que demeure soumise l'activité sportive. Elle n'échappe donc pas à la loi commune. La pratique d'une activité sportive, par essence à risque, est l'occasion de différents préjudices causés par le sportif. Que la victime concernée soit un autre sportif, un spectateur, l'organisateur ou encore l'arbitre notamment, elle invoque régulièrement devant les tribunaux différents arguments juridiques pour obtenir une réparation du préjudice subi. Les responsabilités,

\footnotetext{
${ }^{10}$ La Corruption dans le sport: Une réalité, voir le site internet; https://www.coe.int/t/dg4/epas/Source/Ressources/EPAS_INFO_Bures_fr.pdf; 11/12/2015.

${ }^{11}$ SCHILD, Antrenörün, Hakemin ve Seyircinin Ceza Sorumluluğu, pp. 41-43

${ }^{12}$ LAPOUBLE Jean-Christophe, "Droit du Sport”, L.G.D.J., p. 188.

${ }^{13}$ LAPOUBLE; "Droit du Sport”, p. 183.

${ }^{14}$ HOTIN, Sefkat; "La responsabilité pénale des sportifs", in in Aspects pénaux du droit du sport, Collection CIES, Staempfli Editions SA Berne.2002, p.121, (p.117-134).
} 
qu'elles soient civile ou pénale, le sportif, en lien avec l'activité qu'il exerce, constituent une première source de contentieux. La relation entre le droit de la responsabilité et l'exercice d'une activité physique et sportive ne peut se limiter à l'unique analyse des éléments qui concernent le sportif à l'origine du préjudice qui affecte une tierce personne. Au-delà de cet analyse, d'autres intervenants sont susceptibles d'être directement concernés par une éventuelle mise en oeuvre de leur responsabilité. Tel peut être le cas des organisateurs, des spectateurs ou des arbitres ${ }^{15}$ et plus généralement toutes personnes qui interviennent dans le cadre de l'organisation d'une activité sportive au cours de la quelle un préjudice est causé et doit être réparé. Quelque soit la personne physique ou morale, toute personne est responsable de son acte ${ }^{16}$. Pourtant en droit turc, la personne morale n'est pas responsable pénalement d'après l'article $20 \mathrm{du}$ CPT.

\section{Les Crimes Sportives}

La loi su Sport (6222) de "Lutte contre la violence et le désordre dans le sport" est la base des crimes spécifiques du sport qui date le 14 Avril $2011^{17}$. Cette loi en réalité n'est pas la première loi prévoyant les actes délictuels. Avant cette loi il existait une autre loi de même nom datant 2005 prévoyait des contraventions dont les sanctions étaient administratifs. Mais cette loi n'a pas eu de pouvoir empêcher les faits et le législateur a décidé de faire une autre loi que l'on peut appeler une loi pénale spécifiques aux actes violents ou aux actes qui peuvent provoquer la violence au domain sportif. On peut citer les crimes prévues dans cette présente loi comme; La manipulation du match ( match fixing) ou la trucage, l'injure par la voie de discrimination, vente illégale des billets de compétition, porter des gangs, nuiser les biens etc..La manipulation illégale de compétition sportive était interdite par les réglementations sportives, mais par la loi 6222, elle est acceptée et décrite comme une crime et une sorte de corruption. La peine prévue pour cette crime était de 5 ans jusqu'au 12 ans de prison et une somme de peine pécuniaire. Mais cette peine a été considéré tres lourd et après quelques mois de l'entrée en vigueur de la loi, le législateur a changé la peine et ce qui est aujourd'hui de 1 à 3 ans d'emprison et une somme de peine pécuniaire.

\section{III.CONCLUS $\square$ ON}

En conclusion, la responsabilité pénale se distingue de la reponsabilité civile alors que le responsabilité civile a une vocation réparatrice du dommage causé, au travers de l'allocation de dommages-intérêts, la responsabilité pénale a une fonction exclusivement punitive. Celui qui commet une infraction prend le risque outre l'engagement de sa responsabilité civile, de se voir attribuer une ou plusieurs peines: amende, emprisonnement, confiscation etc. La responsabilité pénale est intimement liée à la commission d'une infraction. La détermination du responsable dépend des règles générales relatives à la responsabilité pénale, de l'identification de celui qui commet les faits répréhensibles et de l'identification des infractions intéressant la pratique du sport comme la violence, le doping et la corruption à part les infractions contre la vie et contre l'integrité du corps humain.

\footnotetext{
${ }_{15}^{15}$ SCHILD, Antrenörün, Hakemin ve Seyircinin Ceza Sorumluluğu, pp. 41-59.

${ }^{16}$ LAPOUBLE; Droit du Sport, pp. 183-185.

${ }^{17}$ DONAY Süheyl, "Sporda Şiddet ve Düzensizliğin Önlenmesi ve Şike ve Teşvik Primi”, 2.ve Güncellenmiş
} Bas1, İstanbul 2012, p.84. 